\title{
Control Chart in Microbiological Cleaning Efficacy of Pharmaceutical Facility
}

\section{Mostafa Essam Eissa, Ahmed Mohamed Mahmoud and Ahmed Saber Nouby}

\author{
Hikma Pharmaceuticals, Egypt
}

Received: November 12, 2015; Accepted: December 13, 2015; Published (web): January 31, 2016

\begin{abstract}
Statistical process control and quality control charts have important applications in the field of microbiological quality control of clean area. Monitoring the environment and the surface samples using contact plates - is critical to verify microbiological cleaning efficacy of the surface and to understand the behavior of the operation. In the current study, RODAC ${ }^{\mathrm{TM}}$ (Replicate Organism Detection and Counting) plates were used to sample surfaces in production rooms for semisolid and liquid products in a course of two years and overall performance was assessed using I-MR chart and data were subjected to analysis. The throughput yield (TPY) was 0.999993 and the overall process capability was acceptable $\left(\mathrm{P}_{\mathrm{pk}}\right.$ was 1.45$)$. The used methodology in microbiological quality control is simple, fast and not expensive, which provides insight for the evaluation of the process efficiency and highlights the limiting factors and the drawbacks that affect the performance.
\end{abstract}

Key words: Quality control charts, microbiological quality control, clean area, environmental monitoring, contact plates, cleaning efficacy, replicate organism detection and counting, throughput yield, process capability

\section{INTRODUCTION}

Monitoring the environment for microorganisms is important in achieving product with compendial requirements. ${ }^{1}$ Regulatory authorities recommend the monitoring of all manufacturing areas to control viable and nonviable particles. Where aseptic operations are performed, monitoring should be frequent, using a variety of methods including contact plates (for work surfaces and personnel monitoring), especially before and after critical operations. $^{2}$ The results should be reviewed and assessed prior to product release. It is important to analyze trends, set appropriate alert and action limits for the monitoring results, and where these limits are exceeded, establish a plan of corrective measures. Surface sampling will indicate the effectiveness of cleaning and disinfection policies. ${ }^{3}$

Correspondence to: Mostafa Essam Eissa

Telephone: 00201006154853

E-mail: mostafaessameissa@yahoo.com

Dhaka Univ. J. Pharm. Sci. 14(2): 133-138, 2015 (December)
The current study is aimed to investigate a methodology for quick and continuous monitoring, follow-up, investigation of the microbiological cleaning efficacy of the surfaces in clean area using Shewhart control chart in conjunction with other tools using commercially available statistical software package.

\section{MATERIALS AND METHODS}

Surface samples using contact plates were taken from pharmaceutical manufacturing plant (liquid oral products and semisolid medicinal drugs: creams, ointments and suppositories area) using methods and limits described by Eissa, 2014. ${ }^{4}$ The total number was 94 different surface samples from class C. All the nutrient media and chemicals were purchased from OXOID (Basingstoke, Hampshire) and SigmaAlrich (St. Louis, MO 63103), respectively. All media were sterilized by autoclaving in steam sterilizer (FEDEGARI FOB3, Fedegari Autoclavi SpA, SS 235 km 8, 27010 Albuzzano (PV), Italy). Contact $55 \mathrm{~mm}$, sterile plates were purchased from 
Sterilin Limited (solaar house, 19 mercer's row, Cambridge, UK). Results of samples were obtained from the microbiology laboratory in the quality control department after incubation in Series BD 115 Incubators with natural convection (BINDER GmbH, Im Mittleren, Ösch 5, 78532 Tuttlingen, Germany). Surface sampling and personnel monitoring activities were performed using contact plates (surface area of approximately $25 \mathrm{~cm}^{2}$ ) filled with an appropriate recovery medium (supplemented with Tween 80 and lecithin). ${ }^{5}$ All statistical analysis and six sigma tools along with their criteria were used as detailed by Eissa et al., 2015. ${ }^{6}$ Culture media used in surface monitoring were subjected to growth promotion tests according to the methods and specifications by USP 2015 and passed the acceptance criteria. ${ }^{7}$ Microbial enumeration was performed using digital colony counter (Digital Colony Counter Model: 361, Laxman Mahtre Rd. Navagaon, Dahisar West, Mumbai). Illustrations of generated data and calculations were performed using Microsoft Office Excel 2007. Pareto charts were constructed using
Minitab $^{\circledR}$ v17.1.0. GraphPad Prism v6.01 for windows was used for statistical analysis.

\section{RESULTS AND DISCUSSION}

Substantial improvements in the microbial quality of manufactured products have been observed in the last decades. Despite these improvements, outbreaks of infection continue to be reported from time to time. ${ }^{8-12}$ Microbial contamination has been one of the prime reasons for regulatory product recalls for the recent years with the most frequently detected Gram-negative organisms. ${ }^{5}$ Hence, strict microbiological quality control (QC) is required to ensure delivery of safe products to the customers and the surface sampling of the clean rooms is an integral part of environmental monitoring (EM) program. Interpretation of the raw data and trending as illustrated in figures 1 and 2 showed that the microbial surface cleanliness assist to improve during the course of two years of the study with the exception of Liquid Filling Material Airlock (LFML),

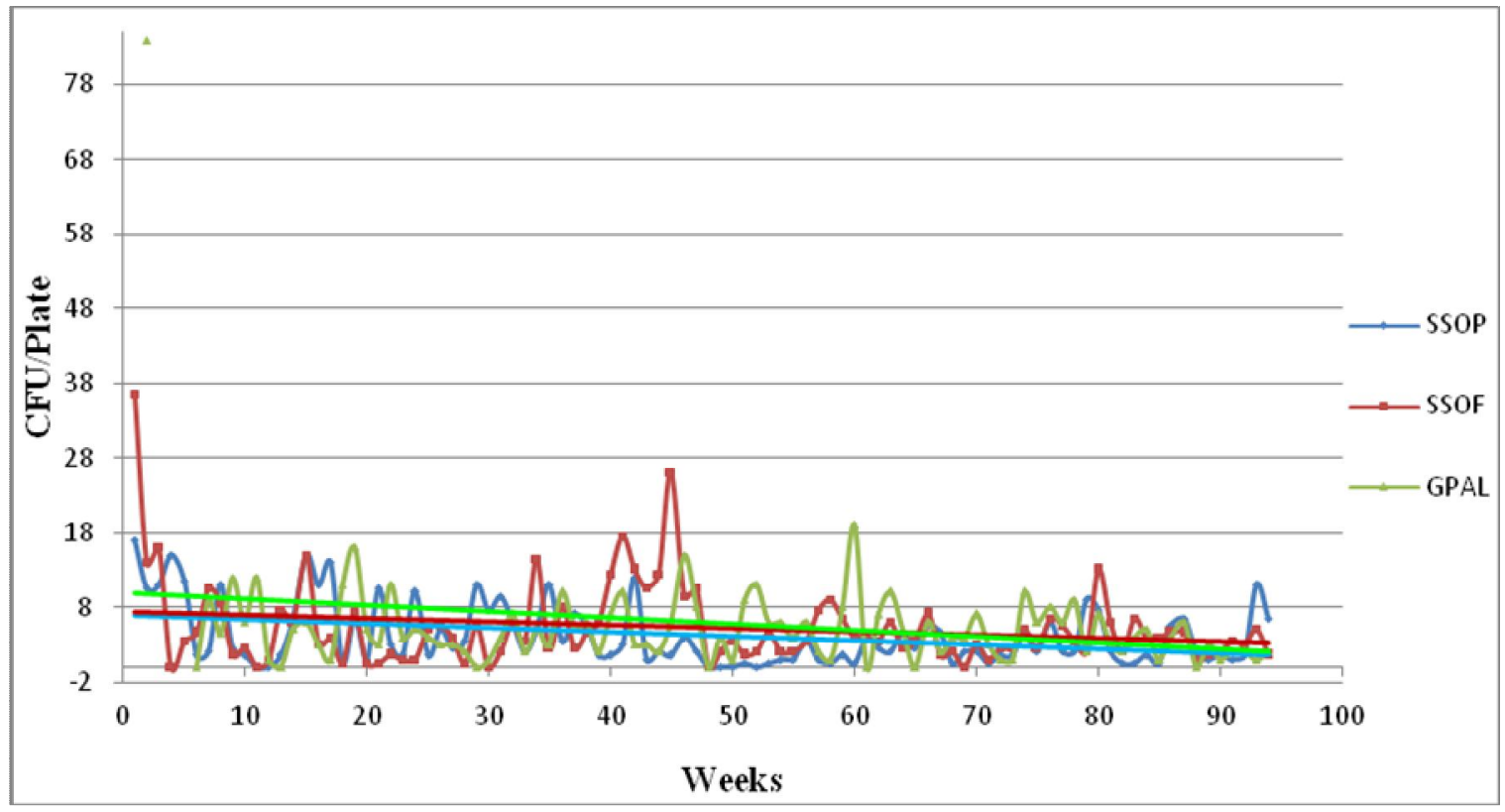




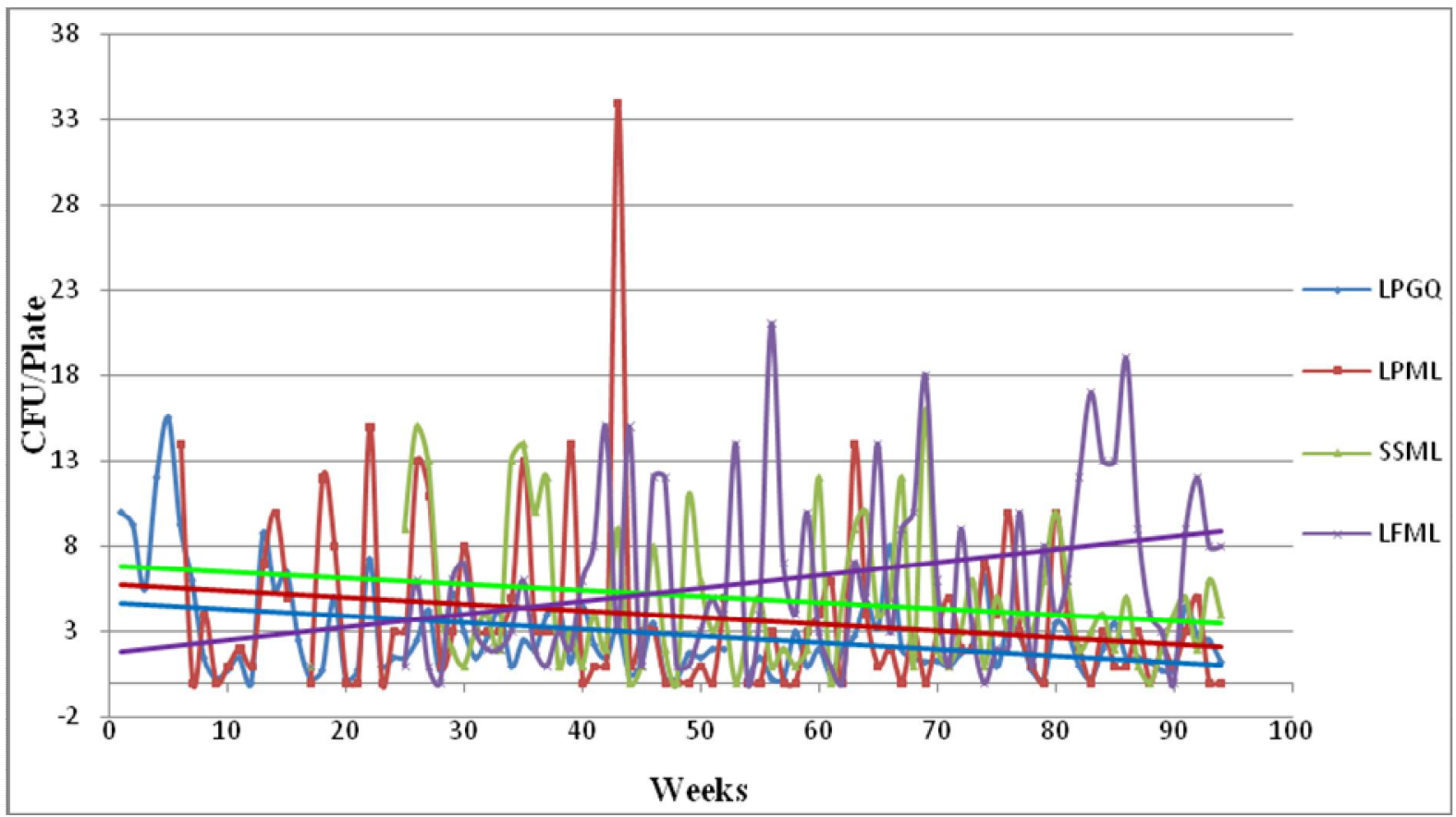

Figure 1. Microbiological surface cleanliness of the manufacturing area: SSOP $=$ Ointment and cream preparation, $\mathrm{SSOF}=$ Ointment and cream filling, GPAL $=$ Personnel airlock, $\mathrm{LPGQ}=$ Liquid preparation, $\mathrm{LPML}=$ Liquid preparation material airlock, SSML $=$ Semisolid material airlock and LFML $=$ Liquid filling material airlock. General trend lines are shown in solid lines (Generated by Microsoft Office Excel, 2007).

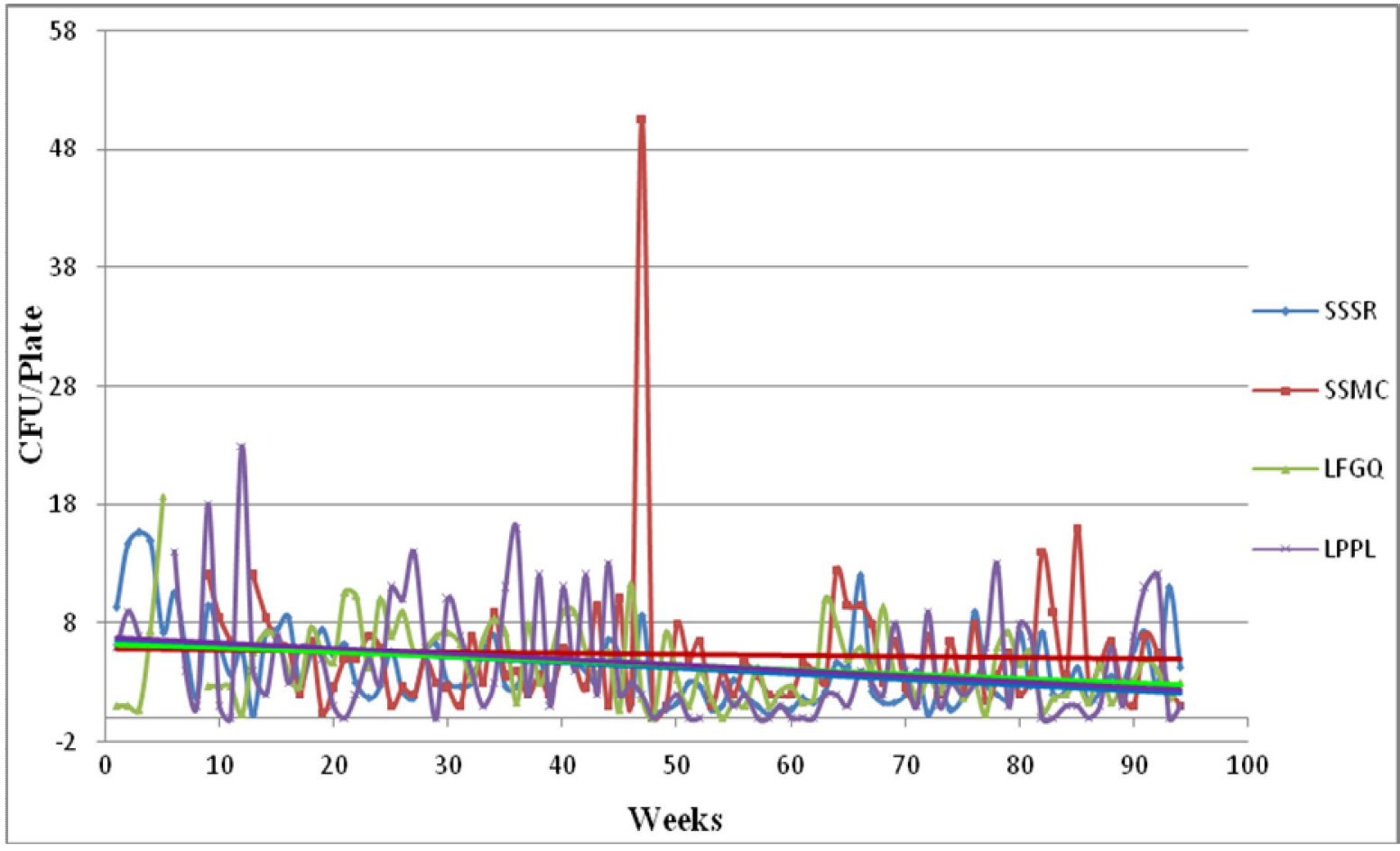




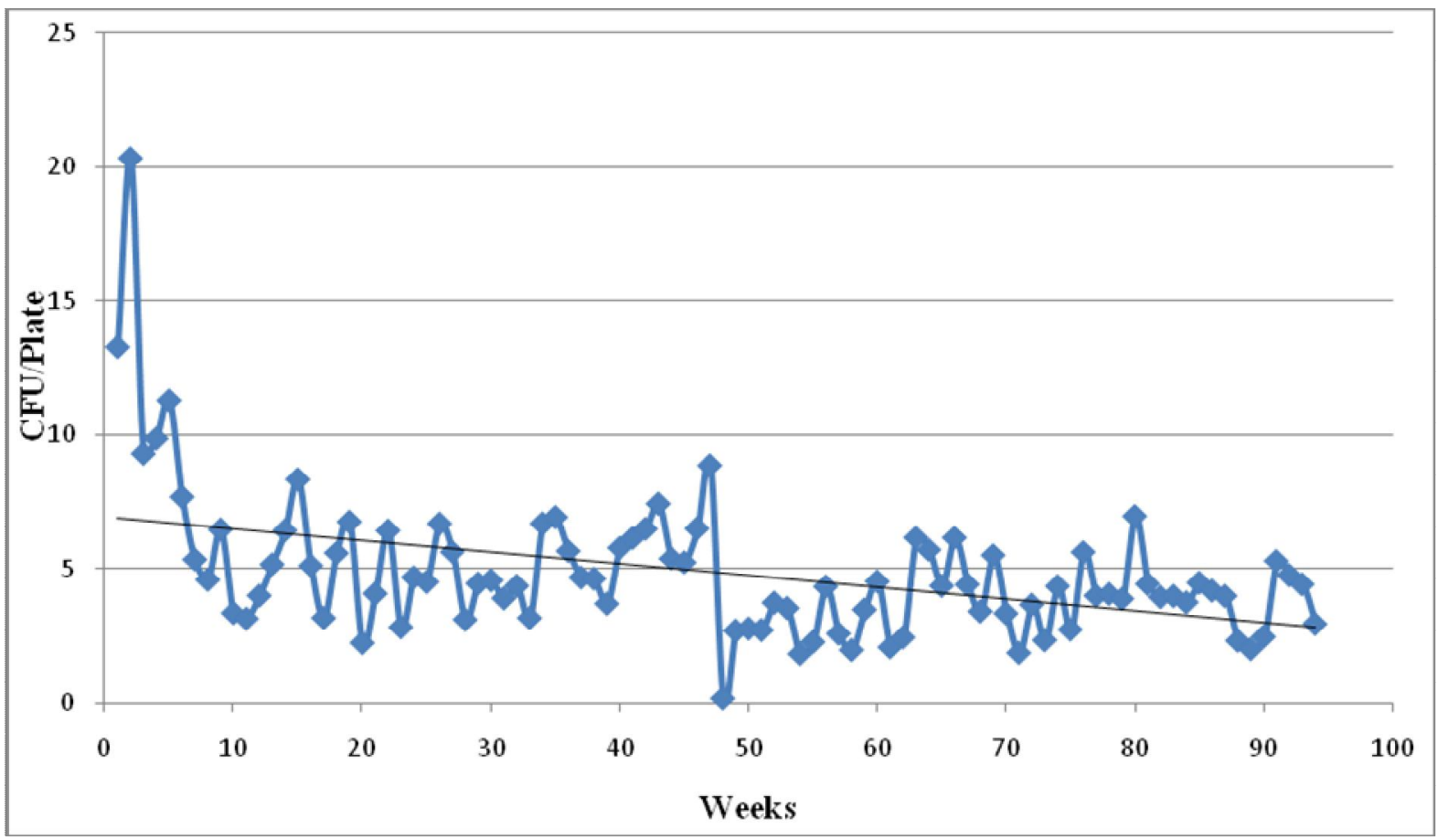

Figure 2. Microbiological surface cleanliness of the manufacturing area: SSSR $=$ Suppositories room, SSMC $=$ Semisolid corridor, $\mathrm{LPPL}=$ Liquid preparation personnel airlock and the lower graph = average trend of class $\mathrm{C}$ during the period of the study. General trend lines are shown in solid lines (Generated by Microsoft Office Excel, 2007).

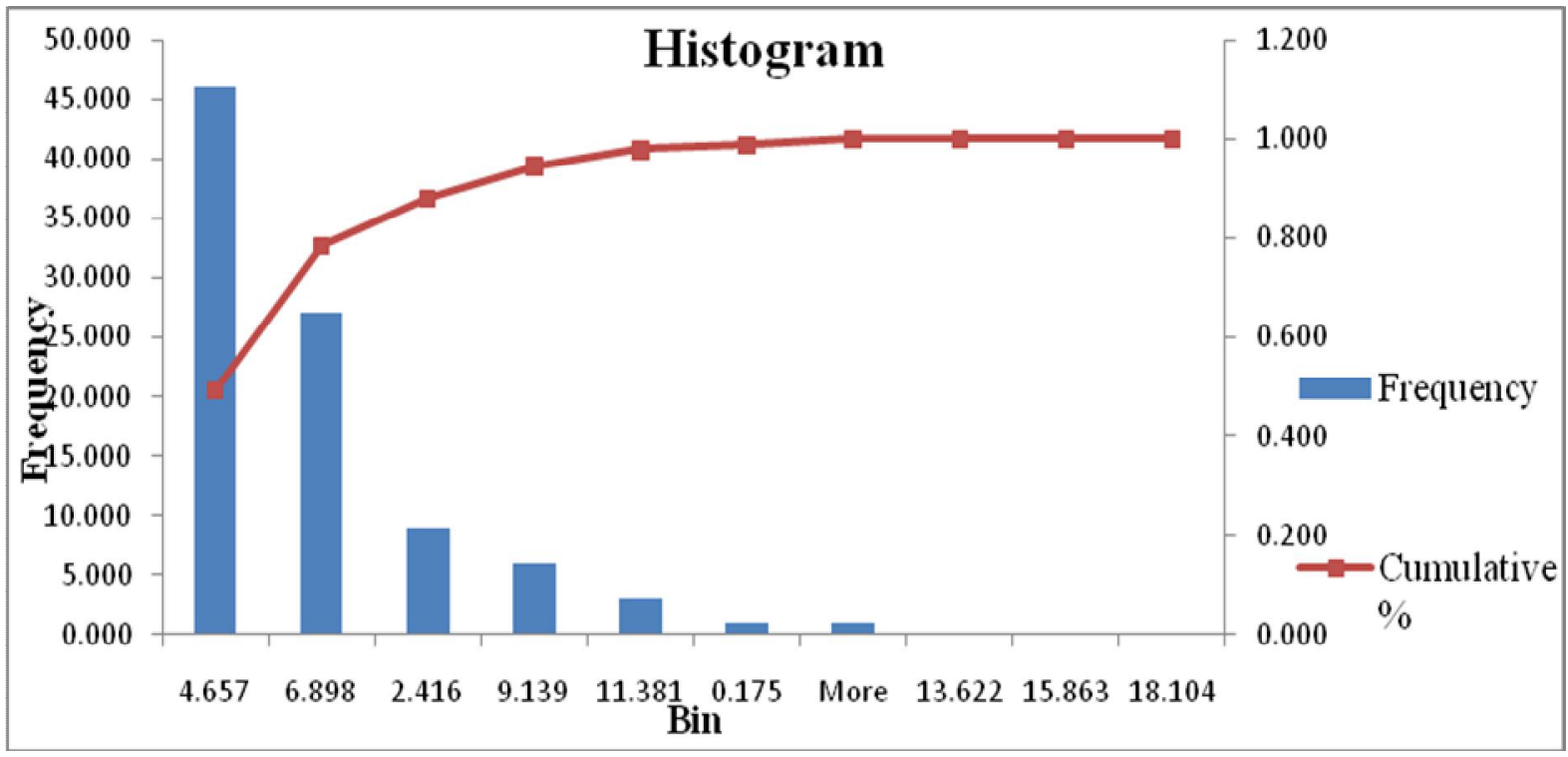

Figure 3. The average microbial count distribution profile on the surfaces of class $\mathrm{C}$ production area for manufacturing of semisolid and liquid medicinal products during a course of two years study. Cumulative data showed that more than $60 \%$ of the results were below five CFU/55 mm diameter contact plate while $89 \%$ of data were below seven CFU/55 mm diameter contact plate (Generated by Microsoft Office Excel, 2007). 


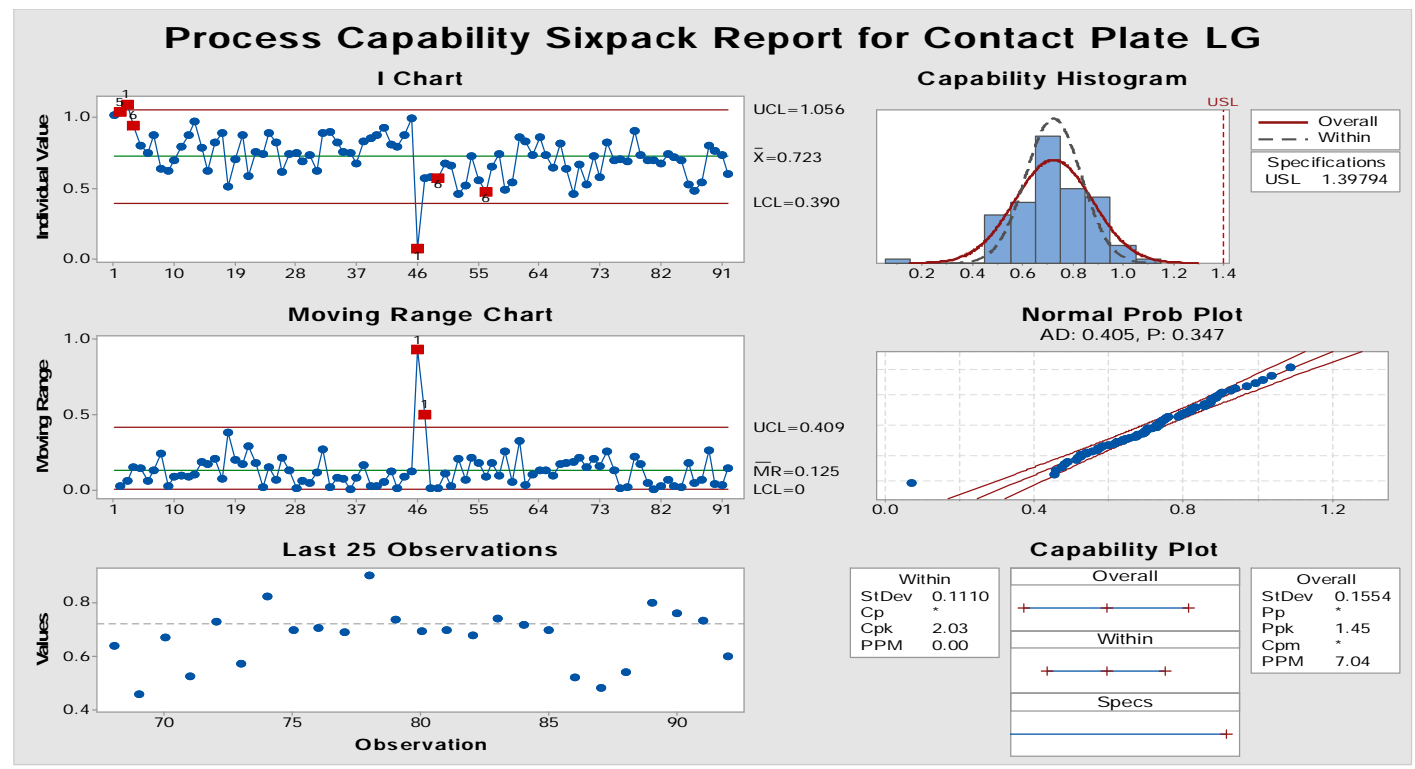

Figure 4. I-MR chart of the averaged logarithmically-transformed to the base ten microbial count results per $55 \mathrm{~mm}$ diameter contact plate from the production area surfaces for verification of microbiological cleanliness and sanitization efficacy of the walls and floors (Generated by using Minitab ${ }^{\circledR}$ v17.1.0).

Where, the results indicated that the cleaning efficacy was continuously deteriorating in figure 1 . Interestingly, it was noted that most excursions in microbial count came from airlocks and corridors where heavy traffic and activities in limited spaces hindered effective sanitization. On the other hand, the histogram of figure 3 for the average results of the surface samples of class $\mathrm{C}$ production area revealed that approximately $95 \%$ of bioburden was below ten CFU/Plate during the course of the study. Logarithmic transformation - to the base ten - of the raw data was the best approach to approximate normality over the other method. This was observed in (Table 1) in which neither original nor square roottransformed results passed the normality test. The constructed I-MR chart in figure 4 demonstrated that the throughput yield (TPY) was 0.999993 and the overall process capability was acceptable $\left(\mathrm{P}_{\mathrm{pk}}\right.$ was 1.45). Excursion was evident only at the initial phase of qualification after which the process was stable and could be described. Since that microbial specification limit (SL) is in one direction only i.e. concerned with the upper specification limit (USL) only but there is no lower specification limit (LSL), the microbiological interest was diverted to the
Table 1. Descriptive statistical analysis of the original and transformed data for the overall averaged microbial count results on the surfaces of the manufacturing facility.

\begin{tabular}{|c|c|c|c|}
\hline Column statistics & $\begin{array}{c}\text { Contact } \\
\text { plate } \\
\text { (raw data) }\end{array}$ & $\begin{array}{l}\text { Contact plate } \\
\text { (square } \\
\text { root) }\end{array}$ & $\begin{array}{c}\text { Contact } \\
\text { plate } \\
\left(\log _{10}\right)\end{array}$ \\
\hline Minimum & 0.0 & 0.42 & 0.070 \\
\hline 25\% Percentile & 3.0 & 1.8 & 0.63 \\
\hline Median & 4.0 & 2.1 & 0.73 \\
\hline $75 \%$ Percentile & 6.0 & 2.4 & 0.83 \\
\hline Maximum & 20 & 4.5 & 1.3 \\
\hline $10 \%$ Percentile & 2.0 & 1.5 & 0.52 \\
\hline $90 \%$ Percentile & 7.0 & 2.7 & 0.91 \\
\hline Mean & 4.8 & 2.1 & 0.73 \\
\hline Std. deviation & 2.6 & 0.54 & 0.17 \\
\hline Std. error of mean & 0.27 & 0.056 & 0.018 \\
\hline Lower $95 \%$ CI of mean & 4.3 & 2.0 & 0.70 \\
\hline Upper $95 \%$ CI of mean & 5.4 & 2.3 & 0.77 \\
\hline Lower $95 \% \mathrm{CI}$ of median & 4.0 & 2.0 & 0.70 \\
\hline Upper $95 \%$ CI of median & 5.0 & 2.2 & 0.75 \\
\hline \multicolumn{4}{|l|}{ KS normality test } \\
\hline KS distance & 0.17 & 0.099 & 0.069 \\
\hline$P$ value & $<0.0001$ & 0.0235 & 0.2000 \\
\hline $\begin{array}{l}\text { Passed normality test } \\
(\text { alpha }=0.05) ?\end{array}$ & No & No & Yes \\
\hline$P$ value summary & $* * * *$ & $*$ & ns \\
\hline Geometric mean & & 2.1 & 0.71 \\
\hline Lower $95 \% \mathrm{CI}$ of geo. mean & & 2.0 & 0.66 \\
\hline Upper $95 \%$ CI of geo. mean & & 2.2 & 0.76 \\
\hline Skewness & 2.5 & 0.93 & -0.013 \\
\hline Kurtosis & 11 & 4.3 & 2.8 \\
\hline Sum & 455 & 201 & 69 \\
\hline
\end{tabular}


out-of-control states above the control limit (CL). Upper control limit (UCL) and CL was approximately $11 \mathrm{CFU}$ and five CFU/Plate respectively. These finding are not strange on the view of other researchers' findings. Microbial burdens of zero to three CFU per $24 \mathrm{~cm}^{2}$ contact plate immediately after cleaning are easily attained and counts of $<10 \mathrm{CFU}$ per $24 \mathrm{~cm}^{2}$ can be expected even during operation. ${ }^{3}$ Probability of defected samples was about seven parts per million (PPM). Normal probability plot confirmed the finding (Table 1) in which transformed data passed normality test.

\section{ACKNOWLEDGEMENTS}

This work was, in part, financially supported by HIKMA Pharmaceutical company. The practical part of all experiments was performed in the microbiology laboratory in the quality control department. Data gathering and issuing were performed by HIKMA microbiology laboratory team.

\section{REFERENCES}

1. Ljungqvist, B. and Reinmüller, B. 1993. Interaction between air movements and the dispersion of contaminants: clean zones with unidirectional air flow. PDA J. Pharm. Sci. Technol. 47, 60-69.

2. Agalloco, J. 1999. Barriers, isolators and microbial control. PDA J. Pharm. Sci. Technol. 53, 48-53.
3. Denyer, S.P. and Rosamund, M.B. 2006. Guide to microbiological control in pharmaceuticals and medical devices. $2^{\text {nd }}$ ed., CRC PR I LIC, New York.

4. Eissa, M.E. 2014. Studies of microbial resistance against some disinfectants: microbial distribution \& biocidal resistance in pharmaceutical manufacturing facility. $1^{\text {st }}$ ed., Saarbrücken, LAP Lamber Academic Publishing.

5. Clontz, L. 2008. Microbial limit and bioburden tests: validation approaches and global requirements. $2^{\text {nd }}$ ed., CRC Press, New York

6. Eissa, M., Seif, M. and Fares, M. 2015. Assessment of purified water quality in pharmaceutical facility using six sigma tools. Int. J. Qual. Assur. 6, 54-72.

7. USP 38 NF 33. 2015. United States Pharmacopeia 38/National Formulary 33, Baltimore, MD, USA.

8. Baird, R.M., Awad, Z.A. and Shooter, R.A. 1980. Contaminated medicaments in use in a hospital for diseases of the skin. J. Hyg. Camb. 84, 103-108.

9. Salveson, A. and Bergen, T. 1981. Contamination of chlorhexidine cream used to prevent ascending urinary tract infections. J. Hyg. Camb. 86, 295-301.

10. Stephenson, J.R., Head, S.R., Richards, M.A. and Tabaqchali, S. 1984. Outbreak of septicaemia due to contaminated mouthwash. Br. Med. 289, 1584.

11. Millership, S.E., Patel, N., Chattopadhyay, B. 1986. The colonization of patients in anintensive treatment unit with Gram-negative flora: the significance of the oral route. $J$. Hosp. Infect. 7, 226-235.

12. Anonymous. 1994. Two children die after receiving infected TPN solutions. Pharm. J. 252, 596. 